Research Paper

\title{
Potential risk of certain cancers among patients with Periodontitis: a supplementary meta-analysis of a large-scale population
}

\author{
Haozhen Ma1,2, Jianmao Zheng ${ }^{1,2}$ and Xiaolan $\mathrm{Li}^{1,2}$ \\ 1. Hospital of Stomatology, Sun Yat-sen University, Guangdong Provincial Key Laboratory of Stomatology, 510055 Guangzhou/PR. China. \\ 2. Guanghua School of Stomatology, Sun Yat-sen University, 510055 Guangzhou/PR. China. \\ $\square$ Corresponding authors: Xiaolan Li, E-mail: lixiaolan@mail.sysu.edu.cn and Jianmao Zheng, E-mail: zhengjm25@mail.sysu.edu.cn.
}

(1) The author(s). This is an open access article distributed under the terms of the Creative Commons Attribution License (https://creativecommons.org/licenses/by/4.0/). See http://ivyspring.com/terms for full terms and conditions.

Received: 2020.04.08; Accepted: 2020.09.03; Published: 2020.09.12

\begin{abstract}
Background: Some studies have reported biological linkages between periodontitis and esophageal cancer, prostate cancer, kidney cancer, hematological malignancy, and melanoma of the skin. This meta-analysis aimed to assess the relationship between periodontitis and the aforementioned five cancers.

Methods: Eligible studies on the association between periodontitis and the aforementioned five kinds of cancers were retrieved. The statistical analysis was conducted using Stata 12.0.

Results: Ten articles (more than 100,000 samples for most cancers) were included. With statistical significance, participants with periodontitis might have enhanced risks of esophageal cancer $(H R=1.79$, $95 \% \mathrm{Cl}: 1.15-2.79)$, prostate cancer $(\mathrm{HR}=1.20,95 \% \mathrm{Cl}: 1.09-1.31)$, hematological malignancy $(\mathrm{HR}=1.19$, 95\% Cl: 1.09-1.29), and melanoma of skin (HR = 1.21, 95\% Cl: 1.03-1.42), compared with those without periodontitis. However, the evidence regarding the correlation between periodontitis and the susceptibility to kidney cancer was lacking $(\mathrm{HR}=1.30,95 \% \mathrm{Cl}: 0.96-1.76)$.

Conclusions: The present meta-analysis revealed a potential link between periodontitis and esophageal cancer, prostate cancer, hematological malignancy, and melanoma of the skin. However, multi-center studies with large sample sizes and multivariable adjustments are still needed to support the conclusion.
\end{abstract}

Key words: Esophageal cancer; prostate cancer; hematological malignancy; melanoma of the skin; periodontitis; meta-analysis

\section{Introduction}

Periodontitis is an inflammatory disease that destroys periodontal tissues (periodontal ligament and alveolar bone). About $10 \%-15 \%$ of the global population suffers from substantial tooth loss caused by severe periodontitis [1]. However, the impact of periodontitis is not confined to oral health. Multiple meta-analyses revealed that participants with periodontitis tended to develop malignant tumors, including oral [2], head and neck [3], lung [4], breast [5], pancreatic [6], and so forth.

However, the correlation between periodontitis and other types of cancers, including esophageal cancer, prostate cancer, kidney cancer, hematological malignancy (HM), and melanoma of the skin, remains unclear. The aforementioned cancers are characterized by high morbidity and fatality rate. According to the GLOBOCAN 2018 database [7], esophageal cancer was the sixth fatal cancer. Prostate cancer was the second most common cancer among men, while renal cancer and melanoma of the skin were the top 20 common cancers. In the USA, HM was the fourth most susceptible cancer, accounting for $9.7 \%$ of all cancers [8]. Therefore, the risk factors of these cancers are needed to be explored to reduce their risk. 
Recently, mounting evidence suggested that periodontitis was potentially associated with esophageal cancer, prostate cancer, kidney cancer, $\mathrm{HM}$, and melanoma of the skin, of which esophageal and prostate cancer were the most studied. Periodontal pathogens with their DNA or virulence factors were detected in esophageal cancer tissues, including Porphyromonas gingivalis (P.gingivalis) [9], Fusobacterium nucleatum (F. nucleatum) [10] and Treponema denticola (T. denticola) [11]. Moreover, P.gingivalis was found to be correlated with the differentiation, metastasis and clinical stage of esophageal cancer. A high level of immune globulins against $P$.gingivalis was related to the poor prognosis of esophageal cancer [12]. As for prostate cancer, scholars found the same pathogens in prostate secretions and dental plaque, including $P$. gingivalis and T. denticola [13]. Furthermore, prostate-specific antigen (PSA, a marker of inflammation or malignancy of the prostate) level was found to be associated with clinical attachment level [14] and periodontal treatment [15]. As for renal cancer, HM, and melanoma of the skin, epidemiological evidences indicated that patients with periodontitis may have enhanced susceptibility to these cancers [16-21]. Besides, associations between periodontitis and some rare subtypes of HM, such as chronic lymphocytic leukemia [22] and lymphoplasmacytic lymphomaWaldenström macroglobulinemia [23], were discovered.

Although there were biologically possible links between periodontitis and the aforementioned five types of malignant tumors, epidemiological researches revealed conflicting results. Due to the lack of statistical effectiveness of a single epidemiological study, this meta-analysis was conducted to provide the latest evidence on the relationship between periodontitis and the aforementioned five cancers.

\section{Methods}

\section{Study retrieval and selection}

Articles published up to August 2020 on the association between periodontitis and the aforementioned five kinds of cancers, were retrieved from PubMed, Web of Science, Embase, and Cochrane Library. The key words were as follows: (1) "esophageal cancer" OR "esophagus tumor" OR "prostate cancer" OR "prostate tumor" OR "kidney cancer" OR "kidney tumor" OR "hematologic malignancy" OR "hematologic cancer" OR "hematologic neoplasm" OR "leukemia" OR "lymphoma" OR "multiple myeloma" OR "melanoma" AND (2) "periodontitis" OR "attachment loss" OR "probing depth" OR "alveolar crestal height". Meanwhile, the references of related studies were also reviewed.

A total of 375 records were obtained, and studies were selected using the following inclusion criteria: (1) studies on human participants; (2) exposure of interest being patients with periodontitis and the control being healthy participants without periodontitis; (3) result of concern being the incidence of esophageal cancer, prostate cancer, kidney cancer, $\mathrm{HM}$, or melanoma of the skin; (4) studies designed as cohort studies, with the full text available; and (5) adjusted hazard ratio (HR) and 95\% confidence intervals $(95 \% \mathrm{CI})$ provided and adjusted confounders reported.

The retrieval and selection were done by two independent authors. Ten articles (referring to 8 studies) were finally included. The detailed process of searching and selection was illustrated in Figure 1.

\section{Ascertainment of periodontitis and cancers}

\section{Ascertainment of periodontitis (precise)}

- A diagnosis code for periodontitis (ICD-9-CM: 523.0-523.5) [21, 24];

- At least two teeth with interproximal (between teeth) attachment loss $\geq 4 \mathrm{~mm}$ or at least two teeth with interproximal pocket depth $\geq 5 \mathrm{~mm}$ (guidelines of the $\mathrm{CDC}$ and the American Academy of Periodontology, CDC/AAP) [25];

- Whole mouth mean alveolar crestal height $(\mathrm{ACH})$ $\geq 2 \mathrm{~mm}$, or at least one site $\mathrm{ACH} \geq 4 \mathrm{~mm}(\mathrm{ACH}$ was defined as the distance in millimeters from the cemento-enamel junction to the alveolarcrest) [20];

- $>2$ interproximal sites with attachment loss (AL) $>3 \mathrm{~mm}$, and $>2$ interproximal sites with probing depth (PD) $>4 \mathrm{~mm}$ (not on same tooth) or one site with PD>5 mm [26];

- $>10 \%$ of examined sites(six sites on all teeth) having AL >3 mm [26];

\section{Ascertainment of periodontitis (imprecise)}

- Self-reported teeth mobility (at least half of the teeth had mobility) [27].

\section{Ascertainment of the 5 cancers}

- Diagnosis codes for the 5 cancers, such as ICD-9-CM 150 (esophageal cancer), ICD-10-CM C61 (prostate cancer) [18, 21, 24, 25, 28];

- Records on cancer registries or death certificates where cancer was the underlying cause of death $[27,29]$.

Detailed criteria of classification of periodontitis severity were shown in Table 1. 


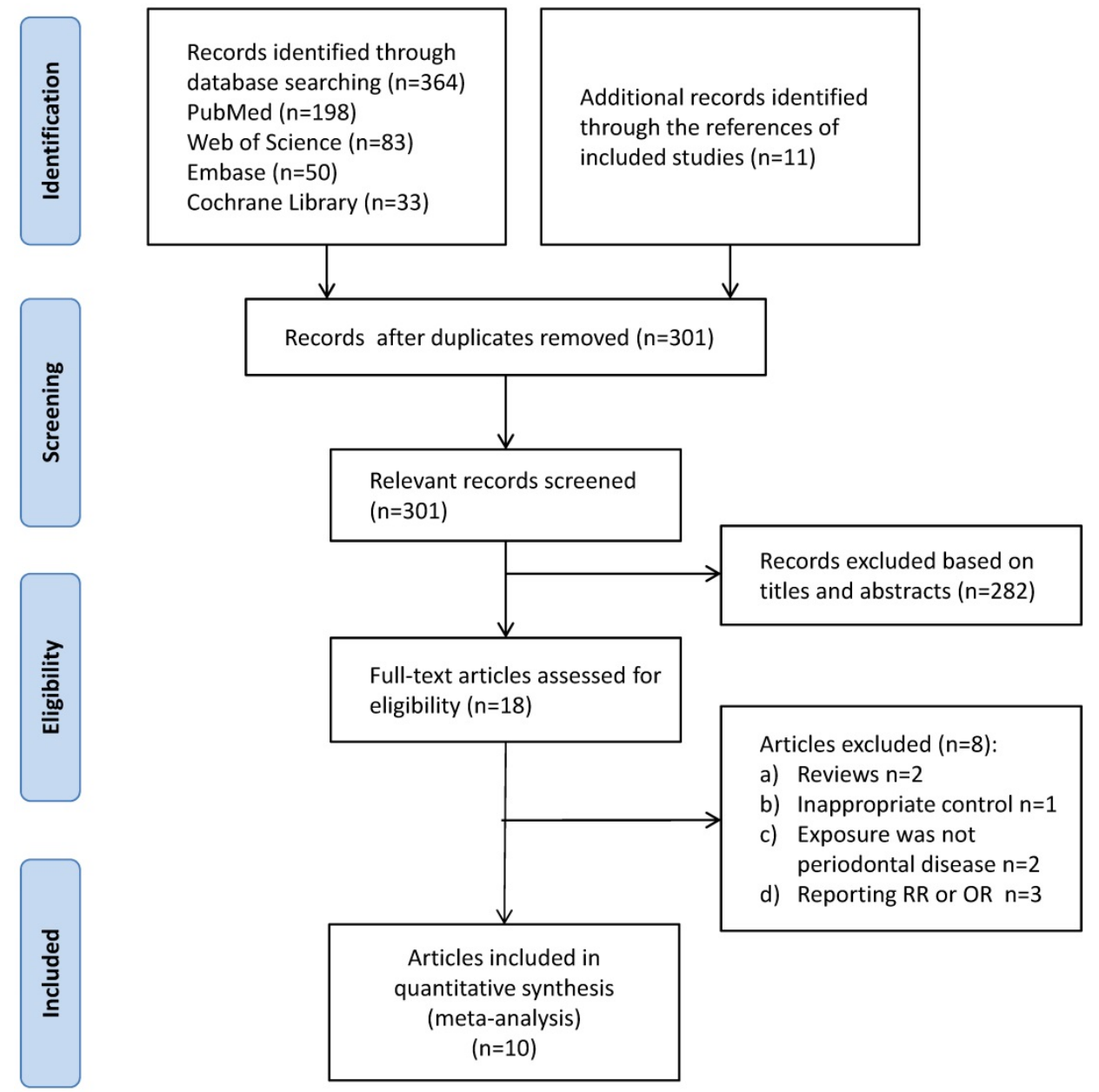

Figure 1. Process of retrieval and selection.

Table 1. Classification of periodontitis severity

\begin{tabular}{|c|c|c|}
\hline & Periodontitis Severity & \\
\hline $\begin{array}{l}\text { Classification } \\
\text { criteria* }\end{array}$ & Mild/Moderate & Severe \\
\hline AL or/and & at least two teeth with & at least two teeth with \\
\hline $\mathrm{PD}[25,26]$ & $\begin{array}{l}\text { interproximal } \mathrm{AL} \geq 4 \mathrm{~mm} \text { or at } \\
\text { least two teeth with } \\
\text { interproximal } \mathrm{PD} \geq 5 \mathrm{~mm}\end{array}$ & $\begin{array}{l}\text { interproximal } \mathrm{AL} \geq 6 \mathrm{~mm} \text { and at } \\
\text { least one tooth with } \\
\text { interproximal } \mathrm{PD} \geq 5 \mathrm{~mm}\end{array}$ \\
\hline AL[26] & $\begin{array}{l}>10 \% \text { to }<30 \% \text { of examined sites } \\
\text { (six sites on all teeth) having } \\
\mathrm{AL}>3 \mathrm{~mm}\end{array}$ & $\begin{array}{l}>30 \% \text { of examined sites (six sites } \\
\text { on all teeth) with } \mathrm{AL}>3 \mathrm{~mm}\end{array}$ \\
\hline $\begin{array}{l}\mathrm{ACH} \text { or/and } \\
\text { tooth loss[20] }\end{array}$ & $\begin{array}{l}\text { whole mouth mean } \mathrm{ACH} \geq 2 \mathrm{~mm} \\
\text { to }<3 \mathrm{~mm} \text {, or at least one site } \\
\mathrm{ACH} \geq 4 \mathrm{~mm} \text {, and no tooth loss } \\
\text { due to periodontitis }\end{array}$ & $\begin{array}{l}\text { whole mouth mean } \mathrm{ACH} \geq 3 \mathrm{~mm} \text {, } \\
\text { or at least two sites } \mathrm{ACH} \geq 5 \mathrm{~mm} \text {, } \\
\text { or tooth loss due to periodontitis }\end{array}$ \\
\hline
\end{tabular}

\section{Information extraction and quality evaluation}

The following information was obtained from 8 involved studies (referring to 10 articles): author, published date, origin of participants, study design, mean follow-up period, mean age, sex, sample size, ascertainment of periodontitis and cancers, severity of periodontitis, cancer types, adjusted covariates and information for quality assessment. Besides, two authors independently evaluated the quality of eligible literatures using the Newcastle-Ottawa scale (NOS). The maximum score could reach 9 points for each study. The specific NOS score was proportional to study quality. NOS scores were divided into three levels: a score of $0-3$, relatively low quality; a score of 4-6, moderate quality; and a score of 7-9, relatively high quality. Tables 2 and 3 illustrated the extracted data and NOS scores. The risk of bias was evaluated by two authors based on a scale in Review Manager 5.2, which included evaluation for cohort design, blinding, missing results, incomplete reporting and so forth. The result was shown in Figure S1 and Figure S2 in the Supplementary File.

\section{Statistical analysis}

We employed the inverse-variance model in Stata 12.0 to obtain overall estimates. HR worked as an indicator to evaluate the correlation between periodontitis and cancers. The heterogeneity was evaluated using $I^{2}$ metric values and their $95 \%$ CIs [30]. The $95 \%$ CIs of $I^{2}$ were calculated based on the classical formulas proposed by Higgins [30]. Randomor fixed-effects models were selected according to 
heterogeneity evaluation. However, if the number of studies on each type of cancer was less than 10 , overall estimates were calculated using random-effects models. Publication bias assessments were conducted through funnel plots. However, funnel plots could not be applied if fewer than 10 studies were included, according to the Cochrane handbook [31]. Stratified by different characteristics of the involved studies, subgroup analyses were used to explore whether the study characteristics influenced the pooled results. Sensitivity analyses were carried out for the evaluations of results' robustness by individually omitting one study at a time. For some types of cancers with few related studies, the sensitivity and subgroup analyses might be waived.

\section{Results}

The included studies were pooled and analyzed by different cancers, as illustrated in Table 4 . Overall estimates were calculated employing random-effects models because of the insufficient number of studies
( $<10$ studies) on each type of cancer. With statistical significance, participants with periodontitis might have enhanced risks of esophageal cancer, prostate cancer, hematological malignancy, and melanoma of the skin, compared with those without periodontitis. However, the evidence regarding the correlation between periodontitis and the susceptibility to kidney cancer was lacking.

\section{Esophageal cancer}

Among all the included studies, four focused on periodontitis and the risk of esophageal cancer $(845,258$ participants in total) $[17,18,24,25]$. The results showed that the exposure to periodontitis might enhance the susceptibility to esophageal carcinoma $\left(\mathrm{HR}=1.79,95 \% \mathrm{CI}: 1.15-2.79, P=0.009, I^{2}=\right.$ $60 \%, \mathrm{P}$ for heterogeneity $=0.056$, Figure 2A). As shown in Figure 2B, the pooled results remained robust when individually leaving out one study at a time. Funnel plot was not applied because of the small number of included studies ( $<10$ studies).

Table 2. Summary of the enrolled studies

\begin{tabular}{|c|c|c|c|c|c|c|c|c|c|c|c|c|}
\hline $\begin{array}{l}\text { Research } \\
\text { ID }\end{array}$ & $\begin{array}{l}\text { Author } \\
\text { year }\end{array}$ & Origin & Study design & $\begin{array}{l}\text { Mean follow- } \\
\text { up period (yrs) }\end{array}$ & $\begin{array}{l}\text { Mean } \\
\text { age }\end{array}$ & Sex & $\begin{array}{l}\text { Sample } \\
\text { size }\end{array}$ & $\begin{array}{l}\text { Periodontitis } \\
\text { assessment }\end{array}$ & $\begin{array}{l}\text { Severity of } \\
\text { periodontitis }\end{array}$ & $\begin{array}{l}\text { Cancer } \\
\text { assessment }\end{array}$ & Cancer types & $\begin{array}{l}\text { NOS } \\
\text { scores }\end{array}$ \\
\hline \multirow[t]{2}{*}{$\# 1$} & $\begin{array}{l}\text { Chung } \\
\text { 2016[21] }\end{array}$ & China & $\begin{array}{l}\text { Retrospective } \\
\text { cohort }\end{array}$ & 5 & 54.1 & Both & 80,280 & $\begin{array}{l}\text { At least two } \\
\text { consensus } \\
\text { diagnoses }\end{array}$ & Unspecified & $\begin{array}{l}\text { Ambulatory } \\
\text { care claims } \\
\text { code }\end{array}$ & $\begin{array}{l}\text { Hematological } \\
\text { malignancy, } \\
\text { etc. }\end{array}$ & 7 \\
\hline & $\begin{array}{l}\text { Lee } \\
\text { 2014[24] }\end{array}$ & China & $\begin{array}{l}\text { Retrospective } \\
\text { cohort }\end{array}$ & 8.425 & 39.8 & Both & 718,409 & $\begin{array}{l}\text { At least two } \\
\text { consensus } \\
\text { diagnoses }\end{array}$ & $\begin{array}{l}\text { Dental prophylaxis } \\
\text { group; Intensive } \\
\text { treatment groupa; } \\
\text { PD without } \\
\text { treatment group. }\end{array}$ & $\begin{array}{l}\text { Ambulatory } \\
\text { care claims } \\
\text { code }\end{array}$ & Esophagus, etc. & 7 \\
\hline$\# 2$ & $\begin{array}{l}\text { Nwizu } \\
2017[18]\end{array}$ & America & $\begin{array}{l}\text { Prospective } \\
\text { cohort }\end{array}$ & 8.32 & 68.3 & Female & 65,869 & Self-report & Unspecified & Medical records & $\begin{array}{l}\text { Esophagus, } \\
\text { Kidney, } \\
\text { Hematological } \\
\text { malignancy, } \\
\text { Melanoma of } \\
\text { the skin, etc. }\end{array}$ & 6 \\
\hline \#3 & $\begin{array}{l}\text { Michaud } \\
2018[26]\end{array}$ & America & $\begin{array}{l}\text { Prospective } \\
\text { cohort }\end{array}$ & 14.7 & 62.5 & Both & 6,056 & $\begin{array}{l}\text { Clinical } \\
\text { diagnosis }\end{array}$ & $\begin{array}{l}\text { No/mild; Moderate; } \\
\text { Severe }\end{array}$ & $\begin{array}{l}\text { State cancer } \\
\text { registries }\end{array}$ & $\begin{array}{l}\text { Prostate, } \\
\text { Hematological } \\
\text { malignancy, } \\
\text { etc. }\end{array}$ & 8 \\
\hline$\# 4$ & $\begin{array}{l}\text { Mai } \\
2016[20]\end{array}$ & America & $\begin{array}{l}\text { Prospective } \\
\text { cohort }\end{array}$ & $12.2 \pm 4.2$ & 66.7 & Female & 1,337 & $\begin{array}{l}\text { Defined by } \\
\text { alveolar crestal } \\
\text { height }\end{array}$ & $\begin{array}{l}\text { Mild/ moderate; } \\
\text { Severe }\end{array}$ & Medical records & $\begin{array}{l}\text { Melanoma of } \\
\text { the skin, } \\
\text { Hematological } \\
\text { malignancy, } \\
\text { etc. }\end{array}$ & 7 \\
\hline \multirow[t]{2}{*}{$\# 5$} & $\begin{array}{l}\text { Michaud } \\
\text { 2008[17] }\end{array}$ & America & $\begin{array}{l}\text { Prospective } \\
\text { cohort }\end{array}$ & 17.7 & 54.42 & Male & 48,375 & $\begin{array}{l}\text { Radiographs } \\
\text { for bone loss } \\
\text { assessment }\end{array}$ & Unspecified & $\begin{array}{l}\text { Medical records } \\
\text { or pathology } \\
\text { reports }\end{array}$ & $\begin{array}{l}\text { Esophagus, } \\
\text { Kidney, } \\
\text { Hematological } \\
\text { malignancy, } \\
\text { etc. }\end{array}$ & 7 \\
\hline & $\begin{array}{l}\text { Michaud } \\
\text { 2016[16] }\end{array}$ & America & $\begin{array}{l}\text { Prospective } \\
\text { cohort }\end{array}$ & 26 & 52.89 & Male & 19,933 & $\begin{array}{l}\text { Radiographs } \\
\text { for bone loss } \\
\text { assessment }\end{array}$ & Unspecified & $\begin{array}{l}\text { Medical records } \\
\text { or pathology } \\
\text { reports }\end{array}$ & $\begin{array}{l}\text { Prostate, } \\
\text { Melanoma of } \\
\text { the skin, etc. }\end{array}$ & 7 \\
\hline \#6 & $\begin{array}{l}\text { Arora } \\
2010[27]\end{array}$ & Sweden & $\begin{array}{l}\text { Prospective } \\
\text { cohort }\end{array}$ & 27 & 51 & Both & 15,333 & $\begin{array}{l}\text { Self-report } \\
\text { teeth mobility }\end{array}$ & $\begin{array}{l}\text { Minor mobility; } \\
\text { Periodontal disease }\end{array}$ & $\begin{array}{l}\text { Records in } \\
\text { cancer register }\end{array}$ & Prostate, etc. & 7 \\
\hline \#7 & $\begin{array}{l}\text { Lee } \\
\text { 2017[28] }\end{array}$ & $\begin{array}{l}\text { South } \\
\text { Korea }\end{array}$ & $\begin{array}{l}\text { Retrospective } \\
\text { cohort }\end{array}$ & 12 & 61.2 & Both & 187,934 & $\begin{array}{l}\text { Clinical and } \\
\text { radiographic } \\
\text { diagnoses }\end{array}$ & Unspecified & $\begin{array}{l}\text { diagnostic } \\
\text { codes }\end{array}$ & Prostate, etc. & 6 \\
\hline$\# 8$ & $\begin{array}{l}\text { Ahn } \\
\text { 2012[25] }\end{array}$ & America & $\begin{array}{l}\text { Prospective } \\
\text { cohort }\end{array}$ & unreported & 38.99 & Both & 12,605 & $\begin{array}{l}\text { Periodontal } \\
\text { attachment loss } \\
\text { and pocket } \\
\text { depth }\end{array}$ & Unspecified & Mortality File & Esophagus, etc. & 6 \\
\hline
\end{tabular}

CI: confidence interval; NOS: Newcastle-Ottawa scale;

a For example, subgingival scaling, gingivectomy, periodontal flap surgery. 


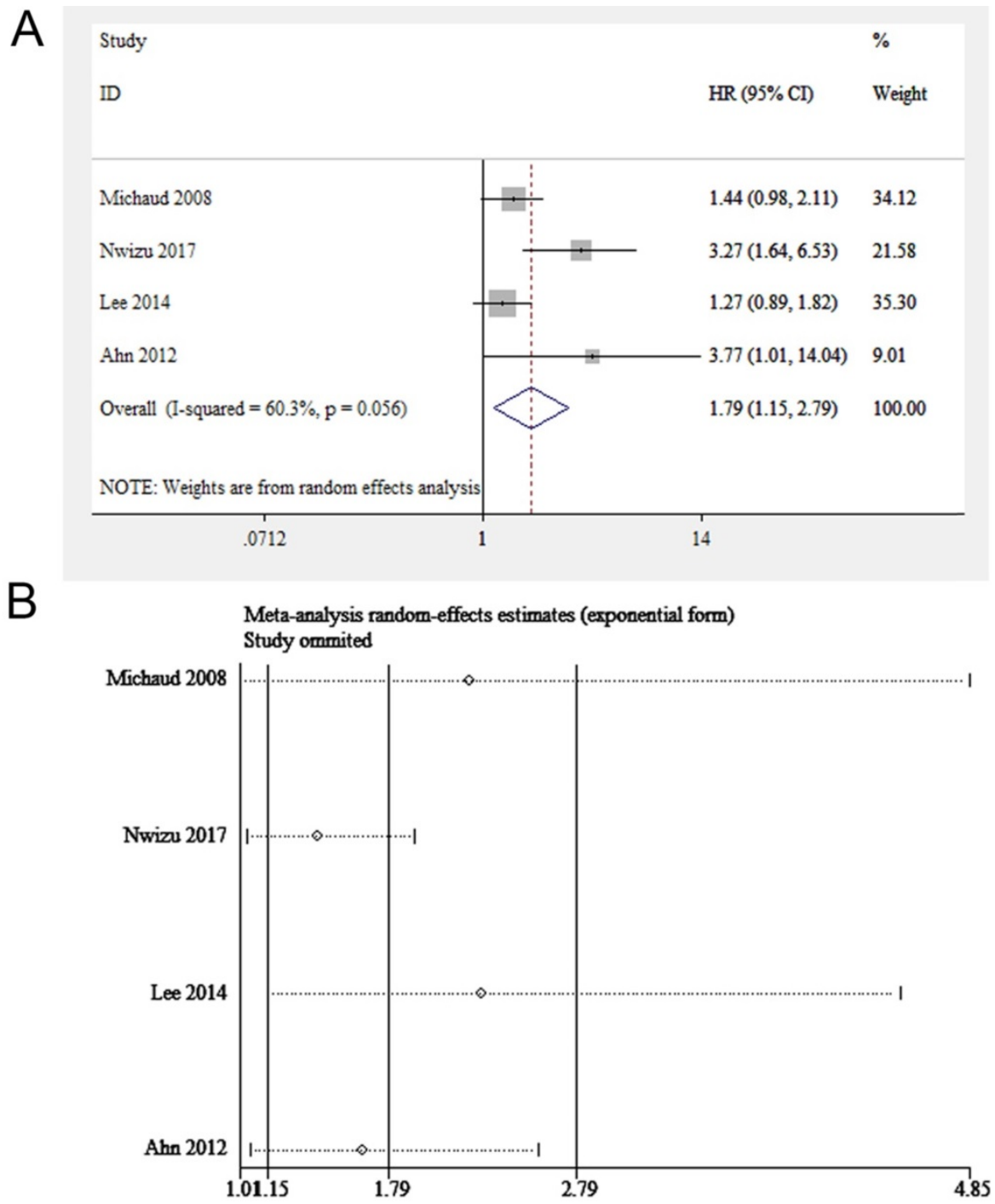

Figure 2. (A) Forest plot and (B) sensitivity analysis of studies on periodontitis and esophageal cancer.

Table 3. Adjusted covariates in studies included in the metaanalysis

\begin{tabular}{|c|c|}
\hline Study & Adjusted covariates \\
\hline Chung 2016 & Age, sex, urbanization, salary, location \\
\hline Lee 2014 & Age, sex, drinking status, comorbidities of esophageal malignancy \\
\hline Nwizu 2017 & $\begin{array}{l}\text { Age, education, geographic location, tobacco consumption, family } \\
\text { history of malignant tumors, exercise habit, secondhand cigarette } \\
\text { smoke, drinking status, fruit and vegetable intake }\end{array}$ \\
\hline Michaud 2018 & $\begin{array}{l}\text { Age, location, educational status, alcohol consumption, blood } \\
\text { glucose level, BMI, race, smoking behaviors }\end{array}$ \\
\hline Mai 2016 & $\begin{array}{l}\text { Age, oral hygiene habits, family history of malignant tumors, } \\
\text { educational status, tobacco consumption, secondhand cigarette } \\
\text { smoke, body mass index }\end{array}$ \\
\hline Michaud 2008 & $\begin{array}{l}\text { Age, frequency of exercise, BMI, energy intake, dietary collocation, } \\
\text { drinking status, tobacco consumption, blood glucose level, smoking } \\
\text { behaviors, calcium supplement, residence, race }\end{array}$ \\
\hline Michaud 2016 & $\begin{array}{l}\text { Age, drinking habit, tobacco consumption, height, frequency of } \\
\text { exercise, BMI, residence region }\end{array}$ \\
\hline Arora 2010 & $\begin{array}{l}\text { Age, gender, blood glucose level, tobacco consumption, tobacco } \\
\text { consumption of partner, drinking behavior, BMI, vocation, } \\
\text { education }\end{array}$ \\
\hline Lee 2017 & $\begin{array}{l}\text { Age, sex, family economic status, location, cardiovascular and } \\
\text { cerebrovascular diseases, physical activity, drinking habit, tobacco } \\
\text { consumption }\end{array}$ \\
\hline Ahn 2012 & Sex, age, ethnic origin, smoking status, education \\
\hline
\end{tabular}

BMI: Body mass index.
Table 4. Results of overall estimates

\begin{tabular}{|c|c|c|c|c|c|c|c|}
\hline \multirow[t]{2}{*}{ Cancer } & \multirow{2}{*}{$\begin{array}{l}\text { No. of } \\
\text { studies }\end{array}$} & \multicolumn{2}{|l|}{ Heterogeneity } & \multirow[t]{2}{*}{ Model } & \multicolumn{3}{|c|}{ Meta-analysis } \\
\hline & & $I^{2}(95 \% \mathrm{CI})$ & $p$ & & HR & $95 \% \mathrm{CI}$ & $p$ \\
\hline Esophageal & 4 & $60 \%(0 \%-87 \%)$ & 0.06 & Random & 1.79 & $1.15-2.79$ & 0.009 \\
\hline Prostate & 4 & $0 \%(0 \%-85 \%)$ & 0.60 & Random & 1.20 & $1.09-1.31$ & 0.0002 \\
\hline Kidney & 2 & $44 \%(0 \%-84 \%)$ & 0.18 & Random & 1.30 & $0.96-1.76$ & 0.09 \\
\hline Hematological & 5 & $0 \%(0 \%-79 \%)$ & 0.61 & Random & 1.19 & $1.09-1.29$ & $<0.0001$ \\
\hline $\begin{array}{l}\text { Melanoma of } \\
\text { the skin }\end{array}$ & 3 & $0 \%(0 \%-90 \%)$ & 0.70 & Random & 1.21 & $1.03-1.42$ & 0.02 \\
\hline
\end{tabular}

Since significant heterogeneity was discovered, a subgroup analysis stratified by sex, age, research quality and adjusted covariates was performed to find out the causes for heterogeneity (Table 5). When stratified by research quality, the value of $I^{2}$ decreased significantly, indicating that the main source of heterogeneity was study quality. As for sex, the heterogeneity of the male group was not significant while that of the female group was obvious, suggesting that sex contributed partly to 
heterogeneity. When pooling the studies with the mean age of participants $\geq 50$, we found periodontitis was potentially linked to esophageal carcinoma $(\mathrm{HR}=2.12$, 95\% CI: 1.01-4.45). When pooling studies with adjusted covariates of smoking or socioeconomic status, we discovered a significant correlation between periodontitis and esophageal carcinoma. None of the four studies had a classification of periodontitis severity; therefore, no subgroup analysis was performed on periodontitis severity.

\section{Prostate cancer}

Four eligible studies (involving 229,256 participants) focused on periodontitis and prostate cancer [16, 26-28]. We found the exposure to periodontitis was potentially linked to prostate cancer $(\mathrm{HR}=1.20,95 \% \mathrm{CI}:$ 1.09-1.31, Figure 3A). The sensitivity analysis illustrated the robustness of the results (Figure 3B). The funnel plot was not used due to the insufficient number of included studies $(<10$ studies).

Table 5. Subgroup analysis of studies on periodontitis and esophageal cancer

\begin{tabular}{|c|c|c|c|c|c|c|c|}
\hline \multirow[t]{2}{*}{ Subgroup } & \multirow{2}{*}{$\begin{array}{l}\text { No. of } \\
\text { studies }\end{array}$} & \multicolumn{2}{|l|}{ Heterogeneity } & \multirow[t]{2}{*}{ Model } & \multicolumn{3}{|c|}{ Meta-analysis } \\
\hline & & $I^{2}(95 \% \mathrm{CI})$ & $p$ & & $\overline{H R}$ & $95 \% \mathrm{CI}$ & $p$ \\
\hline $\begin{array}{l}\text { Overall } \\
\text { analysis }\end{array}$ & 4 & $60 \%(0 \%-87 \%)$ & 0.06 & Random & 1.79 & $1.15-2.79$ & 0.009 \\
\hline \multicolumn{8}{|c|}{ Research quality } \\
\hline Medium & 2 & $0 \%$ & 0.85 & Random & 3.37 & $1.83-6.22$ & $<0.0001$ \\
\hline High & 2 & $0 \%$ & 0.65 & Random & 1.35 & $1.04-1.75$ & 0.03 \\
\hline \multicolumn{8}{|l|}{ Mean age } \\
\hline$<50$ & 2 & $59 \%(0 \%-90 \%)$ & 0.12 & Random & 1.81 & $0.67-4.88$ & 0.24 \\
\hline$\geq 50$ & 2 & $71 \%$ & 0.06 & Random & 2.12 & $1.01-4.45$ & 0.05 \\
\hline \multicolumn{8}{|l|}{ Sex } \\
\hline Male & 2 & $0 \%$ & 0.88 & Random & 1.41 & $1.08-1.83$ & 0.01 \\
\hline Female & 2 & $75 \%(0 \%-94 \%)$ & 0.05 & Random & 1.37 & $0.17-11.02$ & 0.77 \\
\hline \multicolumn{8}{|c|}{ Adjusted covariates } \\
\hline $\begin{array}{l}\text { With smoking } \\
\text { status }\end{array}$ & 3 & $63 \%(0 \%-89 \%)$ & 0.07 & Random & 2.28 & $1.16-4.49$ & 0.02 \\
\hline $\begin{array}{l}\text { With socio- } \\
\text { economic } \\
\text { status }\end{array}$ & 2 & $0 \%$ & 0.85 & Random & 3.37 & $1.83-6.22$ & $<0.0001$ \\
\hline
\end{tabular}
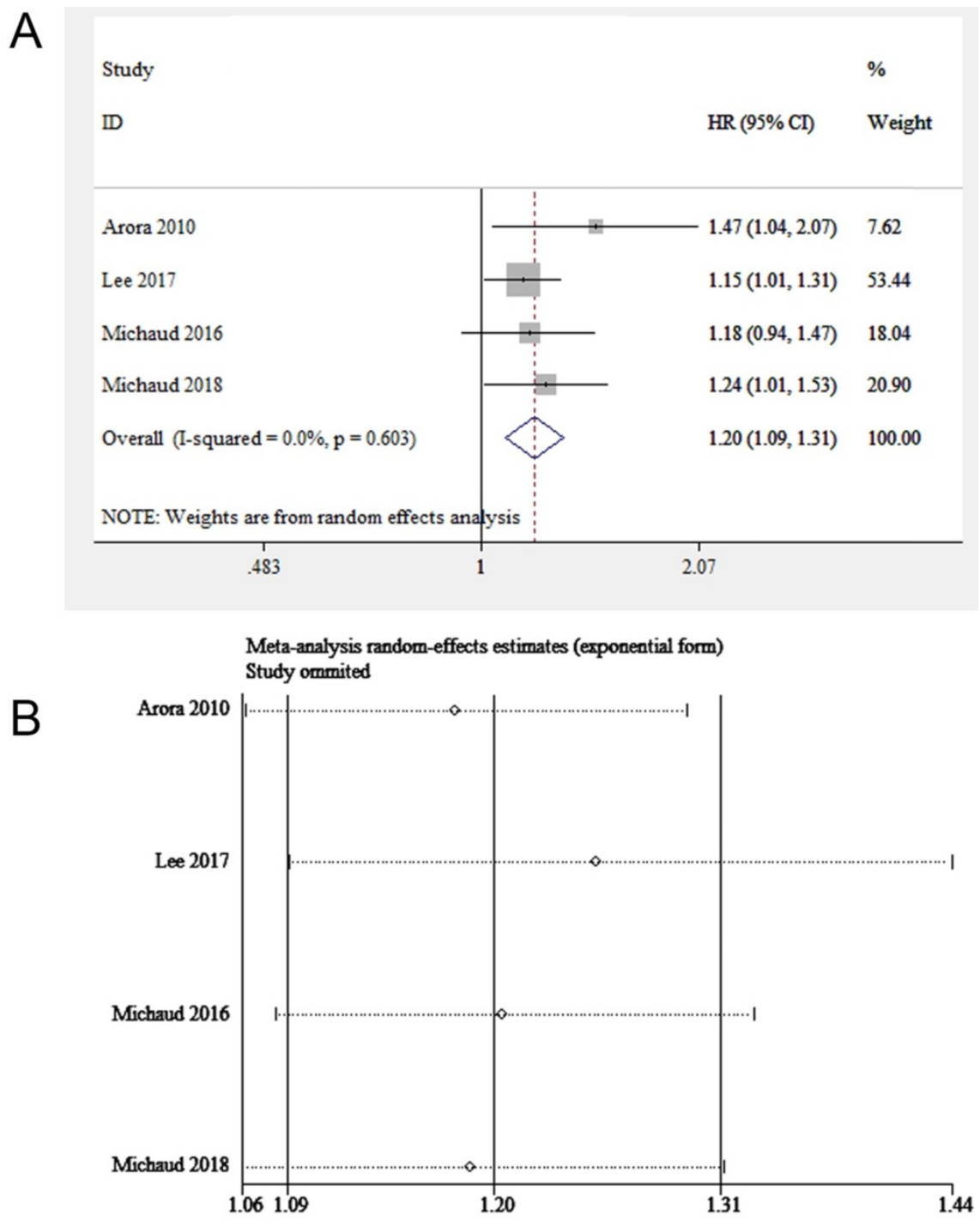

Figure 3. (A) Forest plot and (B) sensitivity analysis of studies on periodontitis and prostate cancer. 
Table 6. Subgroup analysis of studies on periodontitis and prostate cancer

\begin{tabular}{|c|c|c|c|c|c|c|c|}
\hline \multirow[t]{2}{*}{ Subgroup } & \multirow{2}{*}{$\begin{array}{l}\text { No. of } \\
\text { studies }\end{array}$} & \multicolumn{2}{|l|}{ Heterogeneity } & \multirow[t]{2}{*}{ Model } & \multicolumn{3}{|c|}{ Meta-analysis } \\
\hline & & $I^{2}(95 \% \mathrm{CI})$ & $p$ & & HR & $95 \% \mathrm{CI}$ & $p$ \\
\hline \multicolumn{8}{|l|}{$\begin{array}{l}\text { Periodontitis } \\
\text { severity }\end{array}$} \\
\hline $\begin{array}{l}\text { Mild/ } \\
\text { moderate }\end{array}$ & 2 & $0 \%$ & 0.41 & Random & 1.14 & $0.95-1.37$ & 0.17 \\
\hline Severe & 2 & $0 \%$ & 0.48 & Random & 1.34 & $1.06-1.68$ & 0.01 \\
\hline \multicolumn{8}{|l|}{$\begin{array}{l}\text { Adjusted } \\
\text { covariates }\end{array}$} \\
\hline $\begin{array}{l}\text { With smoking } \\
\text { status }\end{array}$ & 4 & $0 \%(0 \%-85 \%)$ & 0.60 & Random & 1.20 & $1.09-1.31$ & 0.002 \\
\hline $\begin{array}{l}\text { With socio- } \\
\text { economic } \\
\text { status }\end{array}$ & 3 & $0 \%(0 \%-58 \%)$ & 0.40 & Random & 1.20 & $1.08-1.33$ & 0.0007 \\
\hline \multicolumn{8}{|l|}{ Mean age } \\
\hline$\leq 60$ & 2 & $\begin{array}{l}11 \% \\
(0 \%-43 \%)\end{array}$ & 0.29 & Random & 1.26 & $1.03-1.54$ & 0.02 \\
\hline$>60$ & 2 & $0 \%$ & 0.53 & Random & 1.18 & $1.05-1.31$ & 0.001 \\
\hline \multicolumn{8}{|l|}{ Sample size } \\
\hline$\leq 15000$ & 2 & $0 \%$ & 0.42 & Random & 1.30 & $1.09-1.55$ & 0.004 \\
\hline$>15000$ & 2 & $0 \%$ & 0.87 & Random & 1.16 & $1.03-1.29$ & 0.01 \\
\hline
\end{tabular}

The subgroup analysis stratified by severity of periodontitis, age, sample size and adjusted covariates was illustrated in Table 6. Among the four studies included, two studies investigated the association between periodontitis of different severity and prostate cancer [26, 27], which were pooled in this subgroup analysis. Mild/Moderate periodontitis might not be related to the enhanced susceptibility to prostate carcinoma, while severe periodontitis was responsible for the enhanced risk of prostatic carcinoma. A correlation between periodontitis and prostate cancer was found when adjusting smoking or socio-economic status. Regarding sample size, a higher risk estimate was obtained from studies with small sample sizes compared to studies with large sample sizes.

\section{Kidney cancer}

Two eligible studies (involving 114,244 participants) focused on correlation between periodontitis and kidney cancer risk [17, 18]. Both of them adjusted important confounders, such as age, race, smoking status, drinking status, fruit and vegetable intake, frequency of exercise and history of diabetes. However, no evident correlation between periodontitis and kidney cancer was found $(\mathrm{HR}=1.30$, 95\% CI: 0.96-1.76, Figure 4).

\section{Hematological malignancy}

Epidemiological studies concerning periodontitis and cancers always combined the cases of leukemia, lymphoma, and multiple myeloma (MM), collectively known as HM. Thus, this study evaluated the relationship between periodontitis and HM, while the respective correlation between periodontitis and leukemia, lymphoma, or MM was assessed in the subgroup analysis. Five eligible studies $[17,18,20,21$, 26] were involved, with 201,917 samples in total. We found periodontitis was potentially associated with $\mathrm{HM}$ risk $(\mathrm{HR}=1.19,95 \% \mathrm{CI}: 1.09-1.29$, Figure 5A). Sensitivity analysis illustrated the homogeneousness and robustness of the results (Figure 5B). Because of insufficient studies involved ( $<10$ studies), the funnel plot was not applied to evaluate publication bias.

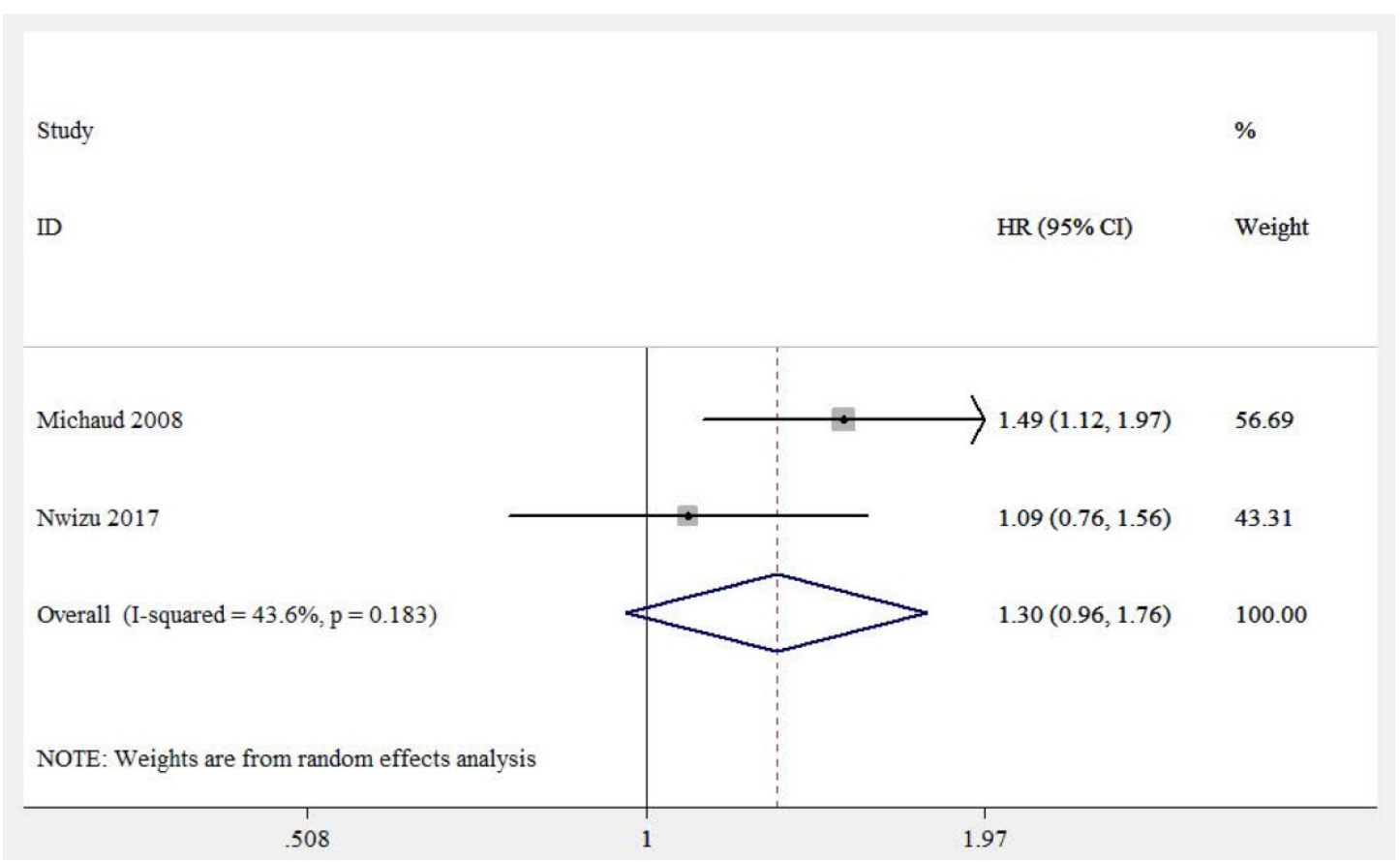

Figure 4. Forest plot of studies on periodontitis and kidney cancer. 

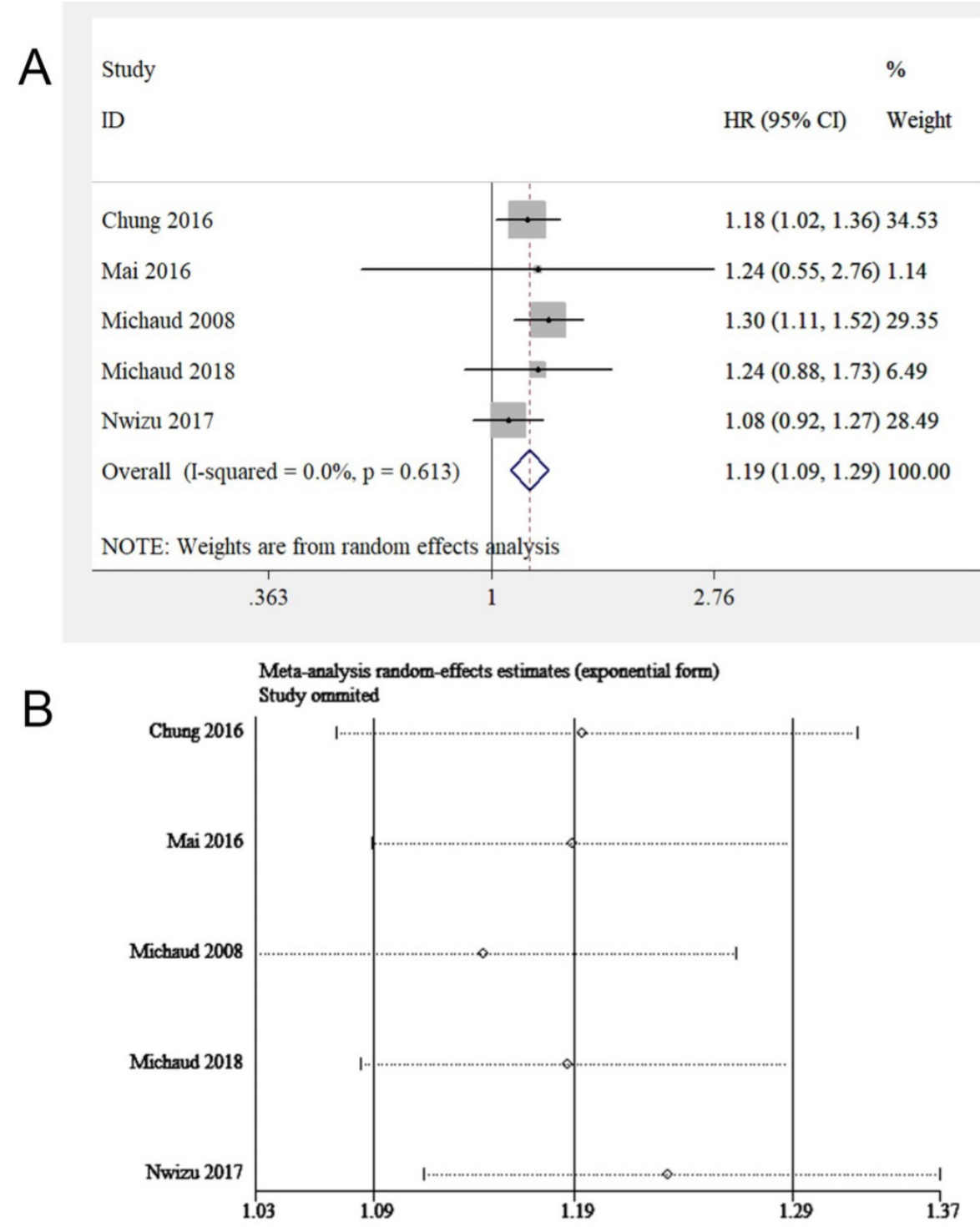

Figure 5. (A) Forest plot and (B) sensitivity analysis of studies on periodontitis and HM.

Subgroup analysis was conducted stratified by periodontitis severity, adjusted covariates, age, sex, follow-up duration, sample size, and HM sub-types (Table 7). A correlation between periodontitis and HM was observed among participants with mean age $<60$ years and male participants. When pooling studies with adjustments on smoking status or socioeconomic factors, a link between periodontitis and HM was discovered. Besides, an association between periodontitis and HM was observed in non-smokers. For the follow-up years, the longer the duration, the higher the HM risk for patients with periodontitis ( $\leq 10$ years: $\mathrm{HR}=1.13,95 \% \mathrm{CI}$ : $1.02-1.26$; $>10$ years: $\mathrm{HR}=1.29,95 \% \mathrm{CI}: 1.12-1.48)$. For HM sub-types, periodontitis enhanced the susceptibility to non-Hodgkin's lymphoma (NHL) $(\mathrm{HR}=1.20,95 \% \mathrm{CI}$ : 1.01-1.43), but the evidence that periodontitis could increase the risk of leukemia and MM was lacking.

\section{Melanoma of the skin}

Three studies, including 87,139 participants, focused on the effect of periodontitis on melanoma of the skin $[16,18,20]$. All of them adjusted important covariates, such as age, educational level and smoking status. By pooling these studies, a potential correlation between periodontitis and melanoma of the skin was discovered ( $\mathrm{HR}=1.21,95 \% \mathrm{CI}: 1.03-1.42$, $I^{2}=0, P$ for heterogeneity $=0.70$, Figure 6A). A sensitivity analysis was subsequently conducted. After excluding Nwizu's study, the overall estimate was no longer statistically significant (Figure 6B). The possible reason was that the sample size of this study accounted for a large part of the total sample size of the included studies. A subgroup analysis was not conducted because of the inclusion of insufficient studies. 
Table 7. Subgroup analysis of studies on periodontitis and HM

\begin{tabular}{|c|c|c|c|c|c|c|c|}
\hline \multirow[t]{2}{*}{ Subgroup } & \multirow[t]{2}{*}{ No. of studies } & \multicolumn{2}{|l|}{ Heterogeneity } & \multirow[t]{2}{*}{ Model } & \multicolumn{3}{|c|}{ Meta-analysis } \\
\hline & & $I^{2}(95 \% \mathrm{CI})$ & $p$ & & HR & $95 \% \mathrm{CI}$ & $p$ \\
\hline Overall analysis & 5 & $0 \%(0 \%-79 \%)$ & 0.61 & Random & 1.19 & $1.09-1.29$ & $<0.0001$ \\
\hline \multicolumn{8}{|l|}{ Mean age } \\
\hline$<60$ & 2 & $0 \%$ & 0.38 & Random & 1.23 & $1.11-1.37$ & 0.0001 \\
\hline$\geq 60$ & 3 & $0 \%(0 \%-90 \%)$ & 0.75 & Random & 1.11 & $0.96-1.28$ & 0.15 \\
\hline \multicolumn{8}{|l|}{ Sex } \\
\hline Male & 2 & $0 \%$ & 0.38 & Random & 1.33 & $1.14-1.54$ & 0.0003 \\
\hline Female & 3 & $0 \%(0 \%-90 \%)$ & 0.51 & Random & 1.09 & $0.93-1.27$ & 0.27 \\
\hline \multicolumn{8}{|l|}{ Periodontitis severity } \\
\hline Mild/moderate & 2 & $0 \%$ & 0.45 & Random & 1.08 & $071-1.65$ & 0.73 \\
\hline Severe & 2 & $0 \%$ & 0.50 & Random & 1.47 & $0.92-2.33$ & 0.11 \\
\hline \multicolumn{8}{|l|}{ Adjusted covariates } \\
\hline With smoking status & 4 & $0 \%(0 \%-85 \%)$ & 0.45 & Random & 1.19 & $1.07-1.33$ & 0.001 \\
\hline With socio-economic status & 4 & $0 \%(0 \%-85 \%)$ & 0.82 & Random & 1.14 & $1.03-1.27$ & 0.009 \\
\hline \multicolumn{8}{|l|}{ Smoking status } \\
\hline Non-smokers & 3 & $0 \%(0 \%-90 \%)$ & 0.63 & Random & 1.30 & $1.14-1.48$ & 0.0001 \\
\hline \multicolumn{8}{|l|}{ Follow-up years } \\
\hline$\leq 10$ & 2 & $0 \%$ & 0.42 & Random & 1.13 & $1.02-1.26$ & 0.02 \\
\hline$>10$ & 3 & $0 \%(0 \%-90 \%)$ & 0.96 & Random & 1.29 & $1.12-1.48$ & 0.0004 \\
\hline \multicolumn{8}{|l|}{ Sample size } \\
\hline$<10000$ & 2 & $0 \%$ & 1.00 & Random & 1.24 & $0.91-1.69$ & 0.18 \\
\hline$\geq 10000$ & 3 & $23 \%(0 \%-70 \%)$ & 0.27 & Random & 1.18 & $1.07-1.31$ & 0.001 \\
\hline \multicolumn{8}{|c|}{ Hematologic cancers sub-types } \\
\hline Leukemia & 2 & $0 \%$ & 0.38 & Random & 1.21 & $0.98-1.49$ & 0.08 \\
\hline Non-Hodgkin lymphoma & 2 & $44 \%(0 \%-85 \%)$ & 0.21 & Random & 1.20 & $1.01-1.43$ & 0.04 \\
\hline Multiple myeloma & 2 & $0 \%$ & 0.44 & Random & 1.17 & $0.89-1.54$ & 0.27 \\
\hline
\end{tabular}

No. of studies: the number of studies.

\section{Discussion}

A consensus about the correlation between periodontitis and five cancers (esophageal cancer, prostate cancer, kidney cancer, HM, and melanoma of the skin) was still lacking, highlighting the necessity for a meta-analysis. This meta-analysis, was performed on 8 studies (10 articles), with a remarkable sample size of more than 100,000 samples for most cancers. By pooling the included studies, a preliminary conclusion was drawn that participants with periodontitis might have enhanced risks of esophageal cancer, prostate cancer, HM, and melanoma of the skin with statistical significance, compared with those without periodontitis. However, no evident correlation between periodontitis and kidney cancer was found. The result of subgroup analysis showed that the periodontitis severity, duration of follow-up, age, sex and the number of participants might influence the result of overall analysis. In addition, observational studies with fewer participants might overestimate the actual correlation, compared with those with more participants. The correlations between periodontitis and two cancers (esophageal cancer and HM) were more significant among male participants than among female participants. Despite the confirmation of the correlation between periodontitis and HM, only the risk of NHL was found to be enhanced when studying the relationship between periodontitis and HM subtypes. The evidence of periodontitis enhancing the susceptibility to leukemia or MM was lacking, which might be attributed to the insufficiency of relevant studies. Therefore, more researches are needed to assess the correlations between periodontitis and HM subtypes.

At present, the exact mechanism of periodontitis promoting esophageal cancer, prostate cancer, HM and melanoma remains unclear. Among these cancers, the mechanisms underlying the relevance between periodontitis and malignant tumors in the esophagus and prostate were studied the most. In esophageal cancer tissues, periodontal microorganisms were found, including P.gingivalis [9], T. denticola [11] and F. nucleatum [10], which might induce inflammation of the esophagus. Cytokines secreted during inflammation, such as interleukin-1 (IL-1) and tumor necrosis factor- $\alpha$ (TNF- $\alpha$ ), as well as reactive oxygen species (ROS), could activate cyclooxygenase 2 (COX-2), which contributed to the development, metastasis, and neo-angiogenesis of esophageal cancer [32]. TNF- $\alpha$ and ROS led to the overexpression of glucose transporters (GLUT-1 and GLUT-4), which were responsible for the nourishment of tumor cells [32]. Pathogens themselves were also related to esophageal carcinogenesis. $T$. denticola could trigger matrix metalloproteinases (MMP-8 and MMP-9) expressions [33], which correlated with immune regulation in tumor tissues [34]. Moreover, an increased MMP-9 level was closely related to poor 
prognosis and metastasis of esophageal tumors [35]. In addition, P.gingivalis could interfere with tumor suppressor p53 and promote carcinogenesis [36]. As for prostate cancer, studies demonstrated a potential link between PSA levels and periodontal conditions [14]. Moreover, periodontal microorganisms similar to those in subgingival plaque were found in prostate secretions [15]. These studies supported the hypothesis that periodontal pathogens and inflammatory substances might migrate through the systemic circulation, causing inflammation of the prostate gland [37, 38]. Local chronic inflammation led to hypoxia, contributing to the release of ROS. This caused COX to convert arachidonic acid into prostaglandins, which regulated cell proliferation [39]. In addition, hypoxia stimulated vascular endothelial growth factor secretion, triggering neo-angiogenesis and fibroblast differentiation, which were promoters of prostatic hyperplasia or tumor [15]. Other studies focused on mechanisms underlying the relevance between periodontitis and HM and melanoma. Studies showed that the citrullination of neutrophils and joint tissue proteins caused by periodontal pathogens (P.gingivalis and Actinobacillus gingivalis) might trigger autoimmune responses [40]. Immune dysfunction, including autoimmune response and chronic inflammation, were risk factors for HM. Therefore, periodontitis might affect the long-term HM risk through autoimmune response or chronic inflammation [41]. Regarding the relevance of periodontitis and cutaneous melanoma, it was discovered that the microbiome (including gut microbiome, oral microbiome and skin microbiome) had a significant impact on the development and immunotherapy of cutaneous melanoma [42-44]. It was speculated that periodontitis might affect the balance of human microbiome and promote the development of melanoma.
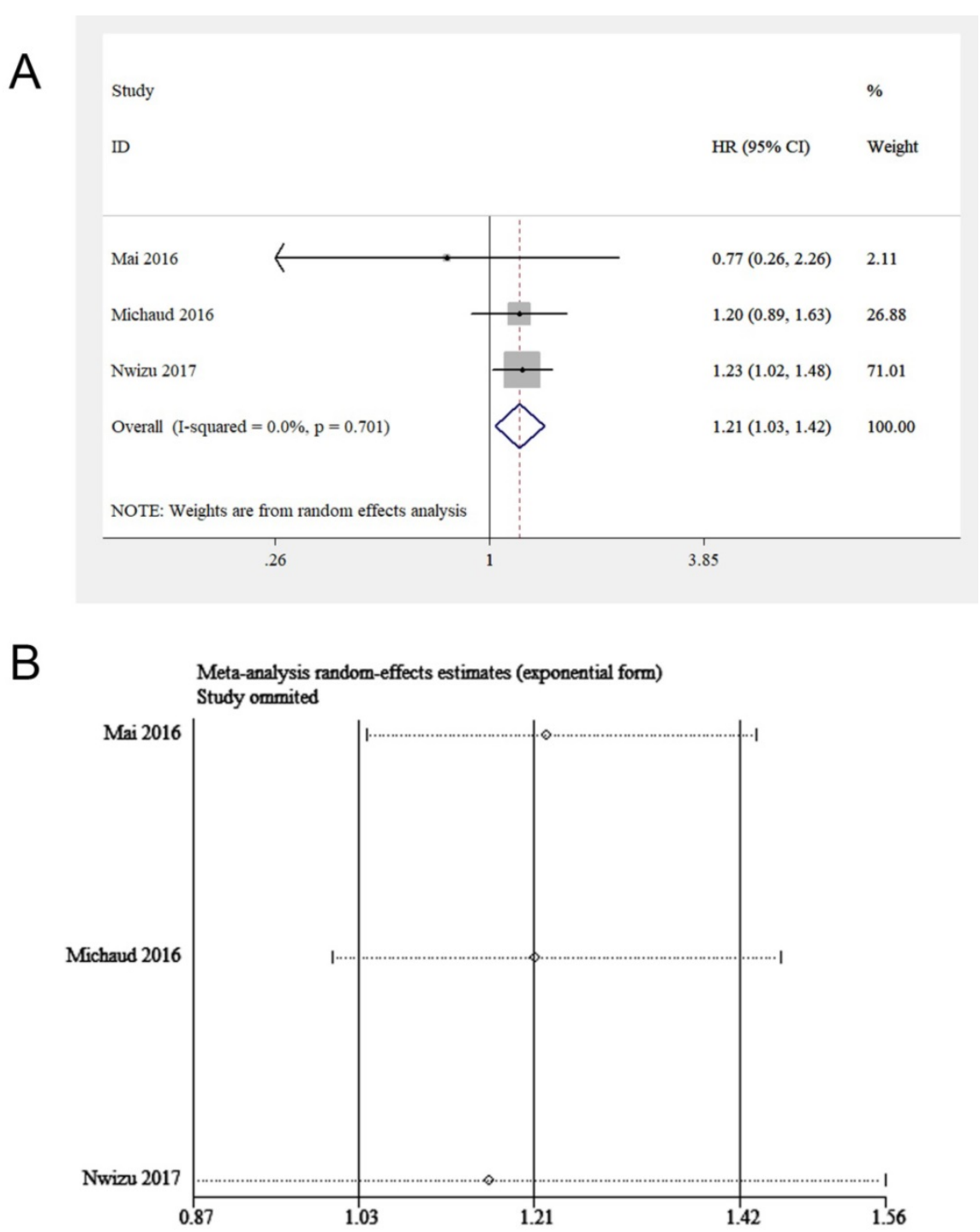

Figure 6. (A) Forest plot and (B) sensitivity analysis of studies on periodontitis and melanoma of the skin. 
However, the aforementioned findings were not sufficient to support a causal relationship between periodontitis and esophageal cancer, prostate cancer, $\mathrm{HM}$ and melanoma of the skin. More researches are needed to reveal the association between periodontitis and the aforementioned cancers.

Age, smoking and socioeconomic status are closely related to the development of cancers. Meanwhile, the aforementioned factors are also significantly related to periodontal conditions. Therefore, the possible influence of the aforementioned confounding factors should be addressed. All of the involved studies adjusted for age. Most studies adjusted for smoking and socioeconomic status. To address possible residual effects caused by smoking, three studies reported analyses restricted to never-smokers [17, 18, 26]. When studying periodontitis and the risk of kidney cancer and melanoma of the skin, all of the included studies adjusted for smoking and socioeconomic status. As for periodontitis and the susceptibility to esophageal cancer, prostate cancer and HM, studies with adjustments of smoking and socioeconomic status were pooled in the subgroup analysis. The resulting HRs were statistically significant, indicating that the association between periodontitis and the aforementioned five cancers was independent of confounders such as age, smoking, and socioeconomic status.

No meta-analysis investigated whether periodontitis increased the risk of esophageal cancer, kidney cancer and melanoma of the skin. Corbella's meta-analysis reported a correlation between periodontitis and prostate tumor (involving 2 studies and 63,708 samples) [45]. Compared with Corbella's meta-analysis, the present meta-analysis had more advantages. First, the results were obtained from a larger database of 4 studies with 229,256 participants. Besides, the present meta-analysis investigated the effect of important confounders in subgroup analysis, such as age, smoking status, socioeconomic status, sample size and periodontitis severity, while Corbella et al. performed no such investigations. Similarly, $\mathrm{Wu}$ 's meta-analysis recently reported a link between periodontitis and HM. [46] However, Wu et al. did not address the possible influence of some significant confounders, such as age and socioeconomic status, which were the risk factors for both periodontitis and cancers. Moreover, they did not calculate the respective risk of leukemia, NHL and MM for patients with periodontitis. On the contrary, the present metaanalysis carefully discussed covariates including age, socioeconomic status, follow-up years, research quality and sample size, in the subgroup analysis. More importantly, it further evaluated the correlation between periodontitis and HM sub-types, including leukemia, lymphoma and MM.

Nevertheless, this study had certain limitations. First, due to the inadequate number of involved literatures, the results obtained were not robust enough. Therefore, it was tentatively suggested that the relevance between periodontitis and the aforementioned cancers was a potential link, not a clear correlation. Second, the confirmation on periodontitis varied from study to study. A majority of included studies were based on standardized dental examinations, while two studies confirming periodontitis based on self-reported tooth mobility $[18,27]$. However, LaMonte et al. [47] assessed the accuracy of self-report. The specificity of selfreporting was up to $94 \%$ [48]. Third, the relevance between periodontitis severity and cancers remained unclear. Some studies were carefully grouped participants according to the severity of periodontitis, but their grouping criteria were not consistent. For each type of cancer, the number of studies stratified by the severity of periodontitis was still insufficient, which might explain why the HRs obtained in the subgroup analysis of periodontitis severity and risk of HM was not statistically significant. Fourth, in subgroup analysis, some of the $95 \%$ CIs of $I^{2}$ were not reported. This was because $95 \%$ CIs of $I^{2}$ cannot be calculated when the number of studies included was insufficient or the value of $\mathrm{Chi}^{2}$ for heterogeneity was too small [30]. Fifth, for kidney cancer, the heterogeneity was significant, which was probably attributed to the insufficiency of included articles. With only two articles involved, the statistical effectiveness of overall estimates for kidney cancer was lacking. Sixth, when assessing the relationship between periodontitis and melanoma of the skin, the exclusion of a study conducted by Nwizu et al. caused a great change in the result [18], which might be attributed to the large sample size of this study and the insufficiency of the number of included studies. Finally, further exploration of the correlation between periodontitis and cancer sub-types was restricted due to insufficient data.

\section{Conclusions}

The present meta-analysis of recent epidemiological studies supported that periodontitis was potentially associated with the development of esophageal cancer, prostate cancer, HM, and melanoma of the skin, highlighting the significance of early prevention and treatment of periodontitis. It must be noted that this meta-analysis was not sufficient to support a causal relationship between periodontitis and the aforementioned cancers. It only revealed a potential link. Moreover, the exact 
mechanism underlying this potential link remained unclear. Multi-center observational studies with an adequate number of participants and multivariable adjustments are required to further assess the correlation between periodontitis and cancers.

\section{Abbreviations}

ACH: Alveolar crestal height; AL: attachment loss; BMI: body mass index; CI: confidence interval; COX: cyclooxygenase; HM: hematological malignancy; HR: hazard ratio; MM: multiple myeloma; NHL: non-Hodgkin's lymphoma; NOS: Newcastle-Ottawa Scale; PD: probing depth; VEGF: vascular endothelial growth factor; ROS: reactive oxygen species.

\section{Supplementary Material}

Supplementary figures.

http://www.medsci.org/v17p2531s1.pdf

\section{Acknowledgements}

This research was funded by the National Natural Science Foundation of China (No. 81700950, 81400505), Guangdong basic and applied basic research Foundation (No. 2019A1515011289), the Youth Teacher Training Project of Sun Yat-Sen University (No. 17ykpy74), and National College Student's Innovation and Entrepreneurship Training Program (No. 20201087). The funding projects had no impact on the design and conduct of the research, and were not involved in result reporting or manuscript submission.

\section{Competing Interests}

The authors have declared that no competing interest exists.

\section{References}

1. Kinane DF, Stathopoulou PG, Papapanou PN. Periodontal diseases. Nature Reviews Disease Primers. 2017; 3: 17038.

2. Ye L, Jiang Y, Liu W, Tao H. Correlation between periodontal disease and oral cancer risk: A meta-analysis. J Cancer Res Ther. 2016; 12: C237-c40.

3. Zeng X-T, Deng A-P, Li C, Xia L-Y, Niu Y-M, Leng W-D. Periodontal disease and risk of head and neck cancer: a meta-analysis of observational studies. PloS one. 2013; 8: e79017-e.

4. Zeng X-T, Xia L-Y, Zhang Y-G, Li S, Leng W-D, Kwong JSW. Periodontal Disease and Incident Lung Cancer Risk: A Meta-Analysis of Cohort Studies. Journal of Periodontology. 2016; 87: 1158-64.

5. Shi T, Min M, Sun C, Zhang Y, Liang M, Sun Y. Periodontal disease and susceptibility to breast cancer: A meta-analysis of observational studies. Journal of clinical periodontology. 2018; 45: 1025-33.

6. Maisonneuve P, Amar S, Lowenfels AB. Periodontal disease, edentulism, and pancreatic cancer: a meta-analysis. Annals of Oncology. 2017; 28: 985-95.

7. Ferlay J, Colombet M, Soerjomataram I, Mathers C, Parkin DM, Piñeros M, et al. Estimating the global cancer incidence and mortality in 2018: GLOBOCAN sources and methods. International Journal of Cancer. 2019; 144: 1941-53.

8. Smith A, Howell D, Patmore R, Jack A, Roman E. Incidence of haematological malignancy by sub-type: a report from the Haematological Malignancy Research Network. British journal of cancer. 2011; 105: 1684.

9. Gao S, Li S, Ma Z, Liang S, Shan T, Zhang M, et al. Presence of Porphyromonas gingivalis in esophagus and its association with the clinicopathological characteristics and survival in patients with esophageal cancer. Infectious agents and cancer. 2016; 11: 3-.
10. Yamamura K, Baba Y, Nakagawa S, Mima K, Miyake K, Nakamura K, et al. Human Microbiome Fusobacterium Nucleatum in Esophageal Cancer Tissue Is Associated with Prognosis. Clinical cancer research : an official journal of the American Association for Cancer Research. 2016; 22: 5574-81.

11. Nieminen MT, Listyarifah D, Hagström J, Haglund C, Grenier D, Nordström $\mathrm{D}$, et al. Treponema denticola chymotrypsin-like proteinase may contribute to orodigestive carcinogenesis through immunomodulation. British journal of cancer. 2018; 118: 428-34

12. Gao S-G, Yang J-Q, Ma Z-K, Yuan X, Zhao C, Wang G-C, et al. Preoperative serum immunoglobulin $\mathrm{G}$ and $\mathrm{A}$ antibodies to Porphyromonas gingivalis are potential serum biomarkers for the diagnosis and prognosis of esophageal squamous cell carcinoma. BMC cancer. 2018; 18: 17-.

13. Estemalik J, Demko C, Bissada NF, Joshi N, Bodner D, Shankar E, et al. Simultaneous Detection of Oral Pathogens in Subgingival Plaque and Prostatic Fluid of Men With Periodontal and Prostatic Diseases. Journal of Periodontology. 2017; 88: 823-9.

14. Joshi N, Bissada NF, Bodner D, MacLennan GT, Narendran S, Jurevic R, et al. Association Between Periodontal Disease and Prostate-Specific Antigen Levels in Chronic Prostatitis Patients. Journal of Periodontology. 2010; 81: 864-9.

15. da Silva APB, Alluri LSC, Bissada NF, Gupta S. Association between oral pathogens and prostate cancer: building the relationship. American journal of clinical and experimental urology. 2019; 7: 1-10.

16. Michaud DS, Kelsey KT, Papathanasiou E, Genco CA, Giovannucci E. Periodontal disease and risk of all cancers among male never smokers: An updated analysis of the Health Professionals Follow-up Study. Annals of Oncology. 2016; 27: 941-7.

17. Michaud DS, Liu Y, Meyer M, Giovannucci E, Joshipura K. Periodontal disease, tooth loss, and cancer risk in male health professionals: a prospective cohort study. The Lancet Oncology. 2008; 9: 550-8.

18. Nwizu NN, Marshall JR, Moysich K, Genco RJ, Hovey KM, Mai X, et al. Periodontal Disease and Incident Cancer Risk among Postmenopausal Women: Results from the Women's Health Initiative Observational Cohort. Cancer epidemiology, biomarkers \& prevention : a publication of the American Association for Cancer Research, cosponsored by the American Society of Preventive Oncology. 2017; 26: 1255-65.

19. Guven DC, Dizdar O, Akman AC, Berker E, Yekeduz E, Ceylan F, et al. Evaluation of cancer risk in patients with periodontal diseases. Turkish journal of medical sciences. 2019; 49: 826-31.

20. Mai X, LaMonte MJ, Hovey KM, Freudenheim JL, Andrews CA, Genco RJ, et al. Periodontal disease severity and cancer risk in postmenopausal women: the Buffalo OsteoPerio Study. Cancer causes \& control : CCC. 2016; 27: 217-28.

21. Chung S-D, Tsai M-C, Huang C-C, Kao L-T, Chen C-H. A population-based study on the associations between chronic periodontitis and the risk of cancer. International journal of clinical oncology. 2016; 21: 219-23.

22. Bertrand KA, Shingala J, Evens A, Birmann BM, Giovannucci E, Michaud DS. Periodontal disease and risk of non-Hodgkin lymphoma in the Health Professionals Follow-Up Study. International Journal of Cancer. 2017; 140: 1020-6.

23. Kristinsson SY, Koshiol J, Björkholm M, Goldin LR, McMaster ML, Turesson I, et al. Immune-Related and Inflammatory Conditions and Risk of Lymphoplasmacytic Lymphoma or Waldenström Macroglobulinemia. JNCI: Journal of the National Cancer Institute. 2010; 102: 557-67.

24. Lee YL, Hu HY, Yang NP, Chou P, Chu D. Dental prophylaxis decreases the risk of esophageal cancer in males; a nationwide population-based study in Taiwan. PloS one. 2014; 9: e109444.

25. Ahn J, Segers S, Hayes RB. Periodontal disease, Porphyromonas gingivalis serum antibody levels and orodigestive cancer mortality. Carcinogenesis. 2012; 33: 1055-8.

26. Michaud DS, Lu J, Peacock-Villada AY, Barber JR, Joshu CE, Prizment AE, et al. Periodontal Disease Assessed Using Clinical Dental Measurements and Cancer Risk in the ARIC Study. Journal of the National Cancer Institute. 2018; 110: 843-54.

27. Arora M, Weuve J, Fall K, Pedersen NL, Mucci LA. An exploration of shared genetic risk factors between periodontal disease and cancers: a prospective co-twin study. American journal of epidemiology. 2010; 171: 253-9.

28. Lee J-H, Kweon HH-I, Choi J-K, Kim Y-T, Choi S-H. Association between Periodontal disease and Prostate cancer: Results of a 12-year Longitudinal Cohort Study in South Korea. J Cancer. 2017; 8: 2959-65.

29. Heikkilä P, But A, Sorsa T, Haukka J. Periodontitis and cancer mortality: Register-based cohort study of 68,273 adults in 10-year follow-up. International Journal of Cancer. 2018; 142: 2244-53.

30. Higgins JP, Thompson SG. Quantifying heterogeneity in a meta-analysis. Stat Med. 2002; 21: 1539-58

31. Cumpston M, Li T, Page MJ, Chandler J, Welch VA, Higgins JP, et al. Updated guidance for trusted systematic reviews: a new edition of the Cochrane Handbook for Systematic Reviews of Interventions. Cochrane Database Syst Rev. 2019; 10: ED000142.

32. Malinowski B, Węsierska A, Zalewska K, Sokołowska MM, Bursiewicz W, Socha M, et al. The role of Tannerella forsythia and Porphyromonas gingivalis in pathogenesis of esophageal cancer. Infectious agents and cancer. 2019; 14: 3-.

33. Yakob M, Meurman JH, Sorsa T, Soder B. Treponema denticola associates with increased levels of MMP-8 and MMP-9 in gingival crevicular fluid. Oral diseases. 2013; 19: 694-701. 
34. Kessenbrock K, Plaks V, Werb Z. Matrix metalloproteinases: regulators of the tumor microenvironment. Cell. 2010; 141: 52-67.

35. Zeng R, Duan L, Kong Y, Liang Y, Wu X, Wei X, et al. Clinicopathological and prognostic role of MMP-9 in esophageal squamous cell carcinoma: a meta-analysis. Chinese journal of cancer research $=$ Chung-kuo yen cheng yen chiu. 2013; 25: 637-45.

36. Kuboniwa M, Hasegawa Y, Mao S, Shizukuishi S, Amano A, Lamont RJ, et al $P$. gingivalis accelerates gingival epithelial cell progression through the cell cycle. Microbes Infect. 2008; 10: 122-8.

37. Van Dyke TE, van Winkelhoff AJ. Infection and inflammatory mechanisms. Journal of clinical periodontology. 2013; 40: S1-S7.

38. Moutsopoulos NM, Madianos PN. Low-Grade Inflammation in Chronic Infectious Diseases. Annals of the New York Academy of Sciences. 2006; 1088: 251-64.

39. Palapattu GS, Sutcliffe S, Bastian PJ, Platz EA, De Marzo AM, Isaacs WB, et al. Prostate carcinogenesis and inflammation: emerging insights. Carcinogenesis. 2005; 26: 1170-81.

40. Konig MF, Abusleme L, Reinholdt J, Palmer RJ, Teles RP, Sampson K, et al. Aggregatibacter actinomycetemcomitans-induced hypercitrullination links periodontal infection to autoimmunity in rheumatoid arthritis. Science translational medicine. 2016; 8: 369ra176.

41. Dhodapkar MV, Dhodapkar KM. Immune Modulation in Hematologic Malignancies. Semin Oncol. 2015; 42: 617-25.

42. Warner AB, McQuade JL. Modifiable Host Factors in Melanoma: Emerging Evidence for Obesity, Diet, Exercise, and the Microbiome. Current Oncology Reports. 2019; 21: 72.

43. Mrázek J, Mekadim C, Kučerová P, Švejstil R, Salmonová H, Vlasáková J, et al. Melanoma-related changes in skin microbiome. Folia Microbiologica. 2019; 64: $435-42$.

44. Gopalakrishnan V, Spencer CN, Nezi L, Reuben A, Andrews MC, Karpinets $\mathrm{TV}$, et al. Gut microbiome modulates response to anti-PD-1 immunotherapy in melanoma patients. Science (New York, NY). 2018; 359: 97-103.

45. Corbella S, Veronesi P, Galimberti V, Weinstein R, Del Fabbro M, Francetti L. Is periodontitis a risk indicator for cancer? A meta-analysis. PloS one. 2018; 13: e0195683-e.

46. Wu Y, Shi X, Li Y, Shi X, Gu Y, Qian Q, et al. Hematopoietic and lymphatic cancers in patients with periodontitis: a systematic review and meta-analysis. Medicina oral, patologia oral y cirugia bucal. 2020; 25: e21-e8.

47. LaMonte MJ, Hovey KM, Millen AE, Genco RJ, Wactawski-Wende J. Accuracy of self-reported periodontal disease in the Women's Health Initiative Observational Study. The Journal of periodontology. 2014; 85: 1006-18.

48. Blicher B, Joshipura K, Eke P. Validation of self-reported periodontal disease: a systematic review. J Dent Res. 2005; 84: 881-90. 